\title{
Galactic Hard X-ray Background: Inner Galaxy
}

\author{
R. Krivonos ${ }^{\star a b \dagger}$ and S. Tsygankov ${ }^{a b c d}$, M. Revnivtsev ${ }^{b}$, S. Sazonov ${ }^{b}$, E. Churazov ${ }^{a b}$ \\ and R. Sunyaev ${ }^{a b}$ \\ ${ }^{a}$ Max-Planck-Institute für Astrophysik, \\ Karl-Schwarzschild-Str. 1, D-85740 Garching bei München,Germany \\ ${ }^{b}$ Space Research Institute, Russian Academy of Sciences, \\ Profsoyuznaya 84/32, 117997 Moscow, Russia \\ ${ }^{c}$ Finnish Centre for Astronomy with ESO (FINCA), University of Turku, \\ Väisäläntie 20, FI-21500 Piikkiö, Finland \\ ${ }^{d}$ Astronomy Division, Department of Physics, FI-90014 University of Oulu, Finland
}

In this report we present measurements of Galactic Ridge X-ray Emission (GRXE) in the Galactic Center region using INTEGRAL scanning observations. The $25-60 \mathrm{keV}$ band GRXE fluxes at $l=0^{\circ}, l=-22.5^{\circ}$, and $l=+22.5^{\circ}$ are $154 \pm 15,72 \pm 15$, and $62 \pm 12 \mathrm{mCrab}$, respectively. The derived longitude profile of the GRXE is in good agreement with the Galactic distribution of stars obtained from infrared observations, which strongly indicates its stellar origin.

The Extreme and Variable High Energy Sky - extremesky2011,

September 19-23, 2011

Chia Laguna (Cagliari), Italy

\footnotetext{
* Speaker.

†E-mail: krivonos@mpa-garching.mpg.de
} 


\section{Introduction}

The stellar origin of the Galactic hard X-ray $(10-100 \mathrm{keV})$ background, better known as the Galactic Ridge X-ray Emission (GRXE), has recently been strongly supported by morphological/spectral studies with the RXTE and INTEGRAL observatories [1, 2, 3], spectral studies with Suzaku [4, 5] and direct X-ray source counts with Chandra [6, 7]. The GRXE does not arise from the interaction of cosmic rays with the interstellar medium, as was believed before, but is associated with the (predominantly old) stellar population of the Galaxy, namely with hard X-ray emission from accreting white dwarfs and coronaly active stars. It was demonstrated $[1,2]$ that GRXE intensity closely follows near-infrared (NIR) surface brightness over the Milky Way, which is a known tracer of stellar mass.

INTEGRAL continues observational campaign for GRXE study. Recent observations in the region of low stellar density at $l=+155^{\circ}$, by Krivonos et al., [8] showed non-detection of the GRXE. It was demonstrated that this result does not contradict the stellar nature of the GRXE, but inconsistent with its cosmic ray origin. In this report we present measurements of the GRXE flux in the central regions of the Galaxy, and compare them with NIR surface brightness and gamma-ray background Galactic longitude profiles.

\section{Observations, analysis and results}

The possibility of the GRXE study with INTEGRAL observatory [9] is based on the capability of the IBIS telescope [10] to separate the contributions of point sources from the background count rate. Large field of view (FOV) of IBIS allows one to collect GRXE flux, but accuracy of its measurement crucially depends on knowledge of instrumental background. In the series of papers $[2,11,8]$ we developed several approaches for ISGRI background modelling for GRXE study with INTEGRAL. The most optimal method includes INTEGRAL scanning observations along the Galactic latitude, when the background model is adjusted very precisely using the mid-latitude snapshots of the background performed shortly before and after a given GRXE observation. This is the main concept of the Galactic Latitude Scans (GLS, [8]). In the present report we use data from the following $G L S$ observations centered at Galactic longitudes

- $l=0^{\circ}$; exposure 1.3 Ms; revolutions 294-296, 357-360, 416-417,

- $l=+22.5^{\circ}$; exposure 1.6 Ms; revolutions 607, 610-615, 667, 670-672, 674, 851-852,

- $l=-22.5^{\circ}$; exposure $630 \mathrm{ks}$; revolutions 1022-1025, 1082-1083.

We screened the whole data set following the procedure described in [2], and built $25-60 \mathrm{keV}$ mosaic sky image of each scan. The list of significantly $(\sigma>5)$ detected sources was used in the iterative procedure of source flux subtraction from the ISGRI detector count rate $[2,11,8]$. The obtained detector light curves sampled per individual INTEGRAL observation with typical exposure of 2 ks (Scientific Window, ScW), and free from source contamination, are shown in Fig. 1.

Using the background model from [8] we obtained for each $S c W$ the predicted detector count rate. As seen from Fig. 1, there is a positive excess between observed and predicted detector 

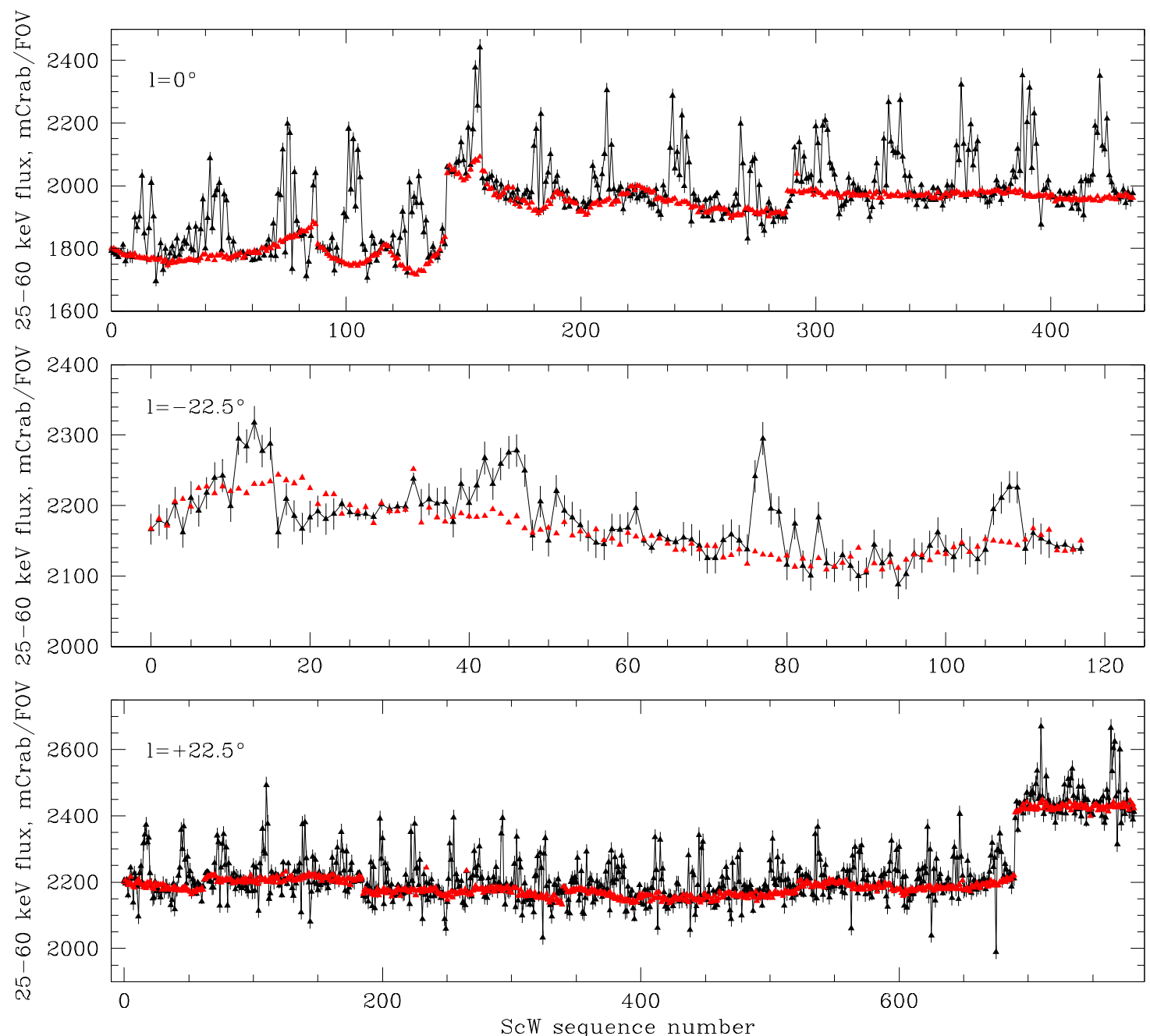

Figure 1: Detector count rate of the individual $S c W s$ as a function of sequence number, after subtracting flux contribution from point sources (in black). The data points contain pure statistical errors. The red points represent the count rate predicted by the background model.

count rate, which is actually the GRXE signal detected when IBIS FOV is crossing the Galactic plane. The sharp changes reflect long-term variability of the background. Averaging this excess over the Galactic latitude we constructed GRXE profiles shown in Fig. 2. All profiles demonstrate significant GRXE detection in the Galactic plane. We measured GRXE flux in the $|b|<5^{\circ}$ latitude range, which roughly corresponds to the IBIS fully coded FOV. Taking systematic uncertainty of $5.7 \mathrm{mCrab}$ in $25-60 \mathrm{keV}$ band ([8]) into account, the obtained flux values are $154 \pm 15,72 \pm 15$, and $62 \pm 12 \mathrm{mCrab}$ at $l=0^{\circ}, l=-22.5^{\circ}$, and $l=+22.5^{\circ}$, respectively. For the sake of convenience of reference, we also show Gaussian functions roughly representing IBIS response function as a collimator. The position, width (FWHM), and normalization are fixed on values $0^{\circ}, 16.45^{\circ}$, and GRXE flux, respectively. GRXE profiles are in general agreement with IBIS response function, however scan at $l=-22.5^{\circ}$, having lower exposure with respect to the others, demonstrate some deviation from Gaussian. The flux measurement at $l=0^{\circ}$ is consistent with $\sim 150$ mCrab GRXE flux in 17-60 keV band obtained in our previous work [2] using the same data set. 

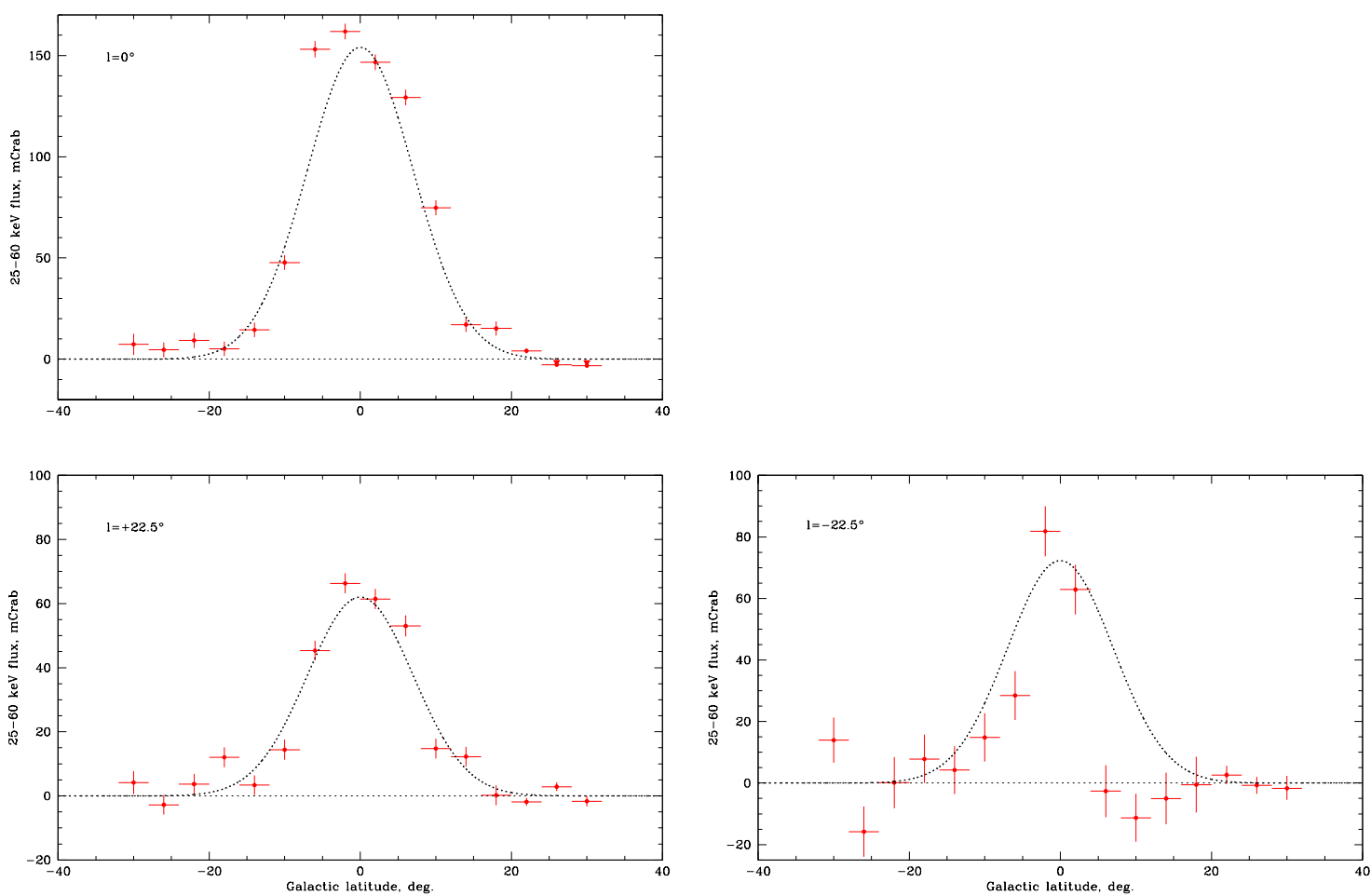

Figure 2: Galactic latitude profiles of the GRXE in the $25-60 \mathrm{keV}$ energy range (in red). Gaussian functions represent IBIS response as a collimated instrument.

We can test the measured fluxes for consistency with stellar and cosmic-ray induced (truly diffuse) GRXE origins. To this end, we compare the observed hard X-ray Galactic longitude profile with the corresponding intensity profiles of a given tracer. We use the COBE/DIRBE $4.9 \mu \mathrm{m}$ data (zodi-subtracted mission average map provided by the LAMBDA archive of the Goddard Space Flight Center, http://lambda.gsfc.nasa.gov, and corrected for the interstellar reddening as described in [2]) as a tracer of stellar mass, and the EGRET gamma-ray background map above $100 \mathrm{MeV}$ as a tracer of the cosmic-ray induced gamma-ray background. This is illustrated in Fig. 3, where the COBE/DIRBE and EGRET longitude profiles (convolved with IBIS response) are renormalized to the hard X-ray flux observed in the GC. The width of INTEGRAL points has been estimated from the effective sensitivity of each data set. We conclude that the measured GRXE fluxes are consistent with the stellar mass distribution of the Galaxy traced by NIR maps, rather than with the cosmic-ray induced gamma-ray background seen by EGRET. This result is in agreement with our previous works $[1,2]$ and supports stellar origin of GRXE.

\section{Acknowledgments}

This research was done thanks to the unique capabilities of the INTEGRAL observatory. The data used were obtained from the European and Russian INTEGRAL Science Data Centers. The work was supported by the President of the Russian Federation (through the program of support of leading scientific schools, project NSH-5069.2010.2, and grant MD-1832.2011.2), by the Presid- 


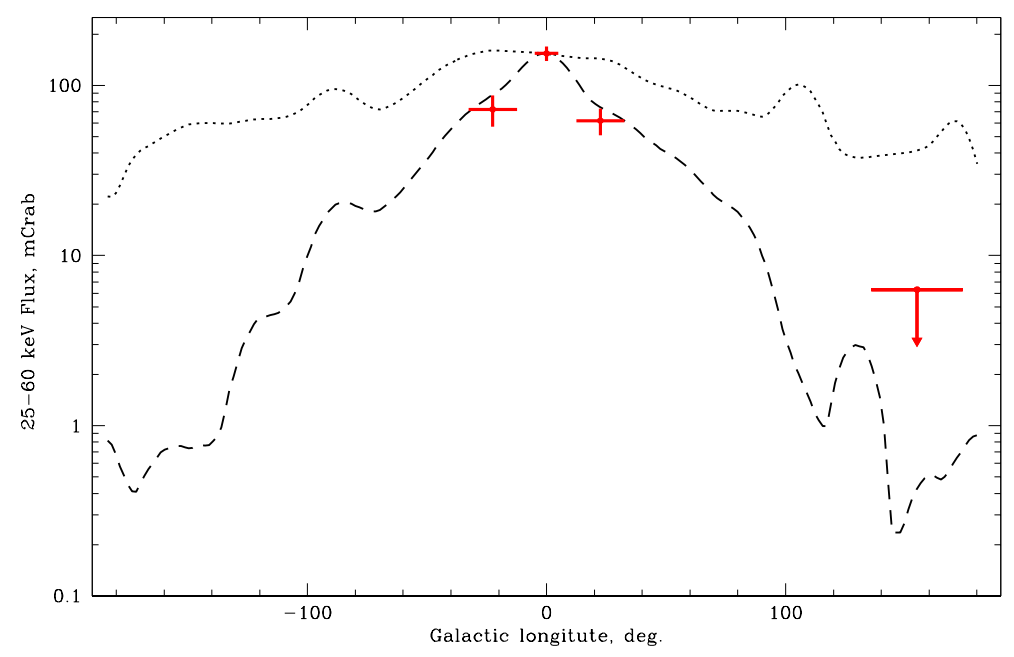

Figure 3: Galactic longitude profiles of the COBE/DIRBE $4.9 \mu \mathrm{m}$ intensity (dashed line) and EGRET background above $100 \mathrm{MeV}$ (dotted line), both normalized to the GRXE flux of $154 \mathrm{mCrab}$ at $l=0^{\circ}$. Red points represent GRXE measurements of the current work. The $2 \sigma$ upper limit corresponds to the GRXE measurement at $l=155^{\circ}[8]$.

ium of the Russian Academy of Sciences/RAS (the program "Origin, Structure, and Evolution of Objects of the Universe"), by the Division of Physical Sciences of the RAS (the program "Extended objects in the Universe", OFN-16), by the Russian Basic Research Foundation (grant 10-02-00492A), State contract 14.740.11.0611, and the Academy of Finland grant 127512.

\section{References}

[1] Revnivtsev, M., Sazonov, S., Gilfanov, M., Churazov, E., \& Sunyaev, R. 2006, A\&A, 452, 169

[2] Krivonos, R., Revnivtsev, M., Churazov, E., Sazonov, S., Grebenev, S., \& Sunyaev, R. 2007, A\&A, 463,957

[3] Türler, M., Chernyakova, M., Courvoisier, T. J.-L., Lubiński, P., Neronov, A., Produit, N., \& Walter, R. 2010, A\&A, 512, A49

[4] Ebisawa, K., et al. 2008, PASJ, 60, 223

[5] Yamauchi, S., Ebisawa, K., Tanaka, Y., Koyama, K., Matsumoto, H., Yamasaki, N. Y., Takahashi, H., \& Ezoe, Y. 2008, arXiv:0810.0317

[6] Revnivtsev, M., Sazonov, S., Churazov, E., Forman, W., Vikhlinin, A., \& Sunyaev, R. 2009, Nature, 458,1142

[7] Revnivtsev, M., Sazonov, S., Forman, W., Churazov, E., \& Sunyaev, R. 2011, MNRAS, 414, 495

[8] Krivonos, R., Tsygankov, S., Revnivtsev, M., et al. 2011, arXiv:1109.2471

[9] Winkler C., et al., 2003, A\&A, 411, L1

[10] Ubertini P., et al., 2003, A\&A, 411, L131 
[11] Krivonos, R., Revnivtsev, M., Tsygankov, S., Sazonov, S., Vikhlinin, A., Pavlinsky, M., Churazov, E., \& Sunyaev, R. 2010, A\&A, 519, A107 